\title{
Saccharomyces: A Friend or Foe in ICU (A Case Report with Solution)
}

\author{
Prasoon Gupta ${ }^{1}$, YP Singh ${ }^{2}$, Akhil Taneja ${ }^{3}$
}

\begin{abstract}
Saccharomyces cerevisiae or boulardii, also known as baker's yeast or brewer's yeast, is normally a nonpathogenic microbe. It is commonly used as a probiotic to prevent antibiotic-associated diarrhea. We present a case of a 77-year-old woman with uncontrolled diabetes who developed Saccharomyces fungemia with use of Saccharomyces containing probiotic after 5 days of treatment. The probiotic was immediately discontinued. The indwelling central line was removed, she was started on amphotericin B and the fungemia resolved. This case report highlights this peculiar complication of probiotic use. We also find it important to increase the awareness amongst the healthcare providers about this likely risk while prescribing probiotics, especially for critically ill patients.

Keywords: Fungemia, Probiotic, Saccharomyces

Indian Journal of Critical Care Medicine (2019): 10.5005/.jp-journals-10071-23239
\end{abstract}

\section{INTRODUCTION}

Yeast are advanced fungi of division Ascomycetes, class Saccharomycetes which grow as single cell and includes Candida and Saccharomyces. 'Saccharomyces and Candida are both a part of the normal flora of airway and gut in humans.

Saccharomyces, is a very common composition of probiotics used in the intensive care unit (ICU) for the treatment of antibioticassociated diarrhea, Clostridium difficile infection and irritable bowel syndrome. ${ }^{2}$ It is claimed to modulate endogenous intestinal flora and the immune system but the evidence in favor of such a claim is very limited. S. boulardii is a subtype of S. cerevisiae, although they are grouped together in the International code of botanical nomenclature $(\mathrm{ICBN})^{3}$ due to similar genetic composition.

\section{Case Description}

A 77-year-old lady, with uncontrolled diabetes (HbA1c-8.4), hypertension and chronic obstructive airway disease, was admitted to the ICU with diagnosed bilateral pneumonia with acute kidney injury. She was intubated in view of respiratory distress and a subclavian central line was inserted to start vasopressors. She was initially managed with injection piperacillin and tazobactum combination along with injection clindamycin. This was later changed to injection meropenem and injection teicoplanin in view of rising leucokyte counts. Subsequently, injection colistin was added, as the culture of endotracheal tube secretions reported carbapenem resistant organism (CRO). Injection Fluconazole was added empirically on day 5 of admission. On day 7, the patient developed watery diarrhea, for which injection racecadotril, injection metronidazole and Saccharomyces containing sachet were added presuming that it was antibiotic induced diarrhea or $C$. difficile infection. Stool routine/microscopy, culture and toxin for $C$. difficile were negative. The diarrhea settled. Three days after starting probiotics, the patient developed hypotension and diarrhea again. Injection fluconazole was changed to injection caspofungin and all cultures were repeated. The initial report of the blood culture after 48 hours were sterile. After four days, blood from central line and peripheral line showed growth of yeast which was reported as
${ }^{1-3}$ Department of Critical Care Medicine, Max Superspeciality Hospital, Patparganj, New Delhi, India

Corresponding Author: Prasoon Gupta, Department of Critical Care Medicine, Max Superspeciality Hospital, Patparganj, New Delhi, India, Phone: 91-9891571699, e-mail: dr.prasoon_gupta@yahoo.com

How to cite this article: Gupta P, Singh YP, Taneja A. Saccharomyces: A Friend or Foe in ICU (A Case Report with Solution). Indian J Crit Care Med 2019;23(9):430-431.

Source of support: Nil

Conflict of interest: None

Saccharomyces cerevisiae (Figs 1 and 2). After a thorough search of literature, we stopped injection caspofungin and started injection amphotericin B. Saccharomyces containing probiotic was stopped and the central line was removed. Repeat blood cultures were sent on the third and seventh day after the positive report; it showed no fungal growth. However, the patient died 24 days post admission.

\section{Discussion}

The first case report of Saccharomyces-related fungemia dates back in 1970 in a patient with prosthetic mitral valve. ${ }^{4}$ Saccharomyces can cause systemic infections like unexplained fever, fungemia, endocarditis, pneumonia, liver abscess, peritonitis and septic shock. ${ }^{5}$ It is very difficult to differentiate this from invasive candidiasis. Fortunately, treatment strategy and antifungal spectrum of both are the same. Risk factors for Saccharomyces fungemia are invasive lines, endotracheal intubation, total parenteral nutrition, immunocompromised host, uncontrolled diabetes, broad-spectrum antibiotics, long hospital stay, cancer, HIV, neutropenia, posttransplant and burns. ${ }^{6}$ The present case was a known case of diabetes, chronic lung disease and was on mechanical ventilation. Management of this condition involves stoppage of probiotic, removal of invasive lines and administration of antifungals. ${ }^{7}$ There is no literature on the antifungal of choice for saccharomyces. Amphotericin B and azoles except itraconazole are preferred along with echinocandins, as demonstrated in a few

(c) The Author(s). 2019 Open Access This article is distributed under the terms of the Creative Commons Attribution 4.0 International License (https://creativecommons. org/licenses/by-nc/4.0/), which permits unrestricted use, distribution, and non-commercial reproduction in any medium, provided you give appropriate credit to the original author(s) and the source, provide a link to the Creative Commons license, and indicate if changes were made. The Creative Commons Public Domain Dedication waiver (http://creativecommons.org/publicdomain/zero/1.0/) applies to the data made available in this article, unless otherwise stated. 
LABORATORY INVESTIGATION REPORT

\begin{tabular}{|llll|}
\hline Patient name: & Mrs. & Location: & Patparganj \\
Age/Sex: & 77 Year(s) Female & IP No./Bed No.: & \\
Maxld: & EHPG & Order Date/Collection Date: & 23/01/2018 / 23/01/2018 07:43 PM \\
Ref. Doctor: & Pulmonology Unit & Report Date: & $30 / 01 / 2018$ \\
\hline
\end{tabular}

Parameter

Sample from:

Comment:

Result:

Comment:

\section{Result}

Central line

Isolated organism - Saccharomyces cerevisiae

Fig. 1: Blood culture and sensitivity from central line showing Saccharomyces cerevisiae

\begin{tabular}{ll}
\hline Antibiotic & Susceptibility \\
\hline Caspofungin & Sensitive \\
Fluconazole & Resistance \\
Flucytosine & Sensitive \\
Micafungin & Sensitive \\
\hline
\end{tabular}

Fig. 2: Saccharomyces antifungal susceptibility

case reports. Combination of amphotericin with flucytosine is used in serious cases. ${ }^{8}$ Even MIC breakpoints of antifungals are not defined by major organizations for Saccharomyces. Two theories are prevalent for Saccharomyces fungemia, first is central line colonization and the other is gut translocation in sick patients. These probiotics are either used as sachets or capsules, in which sachets are more prone to be contaminated by hands of healthcare workers.

Suggested best practices to prevent this complication are:

- Avoid Saccharomyces-based probiotics especially in patients with suspected immune compromise.

- Use capsules and the preparations must be done outside the patient room with change of gloves immediately. ${ }^{9}$

- Lactobacillus-based probiotics can be preferred over Saccharomyces-based probiotics.

- It is important to differentiate between Candida and Saccharomyces early by the microbiologist. It may help them by providing detail, if patient is on Saccharomyces supplement.

- Awareness among clinicians about the risk factors of prescribing probiotics in ICU.

- It is advisable to send catheter tip culture after line removal so that central line or gut translocation as the possible source can be ascertained.

- The manufacturers of these supplements should be directed to have special warning tags about this complication

- More studies to be carried out for echinocandins as antifungal for Saccharomyces.

- MIC breakpoint needs to be defined.

\section{Conclusion}

Saccharomyces based probiotics should be used with caution in critically III patients. If any culture shows budding yeast cell and patient is on Saccharomyces-based probiotics, it is better to have a personal communication with microbiologist. Treatment strategy includes discontinuation of probiotics, removal of central venous catheters and echinocandin or amphotericin-based antifungals.

\section{ACKNOWLedgment}

We acknowledge extreme support of Dr Prakash Shastri for valuable inputs in improving manuscript.

\section{References}

1. Sung-Oui Suh, Meredith Blackwel, Cletus P Kurtzman, Marc-Andre' Lachance. Phylogenetics of Saccharomycetales, the ascomycete yeasts. Mycologia 2006;98(6):1006-1017.

2. Thygesen JB, Glerup H, Tarp B. Saccharomyces boulardii fungemia caused by treatment with a probioticum. BMJ Case Reports 2012;10.1136/bcr.06.2011.4412.

3. I Kara, F Y1ld1r1ma, Ö Özgen, S Erganis, M Aydog ¿du, M Dizbay, et al. Saccharomyces cerevisiae fungemia after probiotic treatment in an intensive care unit patient. Journal de Mycologie Médicale 2018;28:218-221.

4. Stein PD, Folkens AT, Hruska KA. Saccharomyces fungemia. Chest 1970; 58: 173-175.

5. Santino I, Alari A, Bono S, Teti E, Marangi M, Bernardini A, et al. Saccharomyces cerevisiae fungemia, a possible consequence of the treatment of Clostridium difficile colitis with a probioticum. Int Immunopathol Pharmacol 2014;27:143-146.

6. Patricia Munoz, Emilio Bouza, Manuel Cuenca-Estrella, Jose Marı́a Eiros, Maria Jesu's Pe'rez, Maria Sa'nchez-Somolinos, et al. Saccharomyces cerevisiae Fungemia:An Emerging Infectious Disease. Infectious Diseases 2005; 40:1625-1634.

7. Serkan Atıcı, Ahmet Soysal, Kıvılcım Karadeniz Cerit, Şerife Yılmaz, Burak Aksu, Gürsu Kıyan, et al. Catheter-related Saccharomyces cerevisiae Fungemia Following Saccharomyces boulardii Probiotic Treatment: In a child in intensive care unit and review of the literature. Medical Mycology Case Reports 2017;15:33-35.

8. Tiballi RN, Spiegel JE, Zarins LT, Kauffman CA. Saccharomyces cerevisiae infections and antifungal susceptibility studies by colorimetric and broth macrodilution methods. Diagnostic Microbiology and Infectious Disease 1995;23:135-140.

9. Hennequin C, Kauffmann-Lacroix C, Jobert A, Viard JP, Ricour $C$, Jacquemin $\mathrm{JL}$, et al. Possible role of catheters in Saccharomyces boulardii fungemia. Eur J Clin Microbiol Infect Dis. 2000;19(1):16-20. 